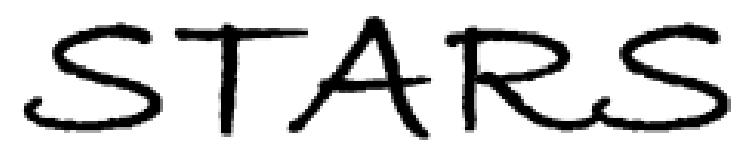

University of Central Florida

STARS

$1-1-1988$

\title{
Crossover Of The Spectral Weight Function: A New Interpretation Of Some Neutron-Scattering Data
}

\author{
Lee Chow \\ University of Central Florida \\ David Fisher \\ University of Central Florida
}

Find similar works at: https://stars.library.ucf.edu/facultybib1980

University of Central Florida Libraries http://library.ucf.edu

This Note is brought to you for free and open access by the Faculty Bibliography at STARS. It has been accepted for inclusion in Faculty Bibliography 1980 s by an authorized administrator of STARS. For more information, please contact STARS@ucf.edu.

\section{Recommended Citation}

Chow, Lee and Fisher, David, "Crossover Of The Spectral Weight Function: A New Interpretation Of Some Neutron-Scattering Data" (1988). Faculty Bibliography 1980s. 605.

https://stars.library.ucf.edu/facultybib1980/605

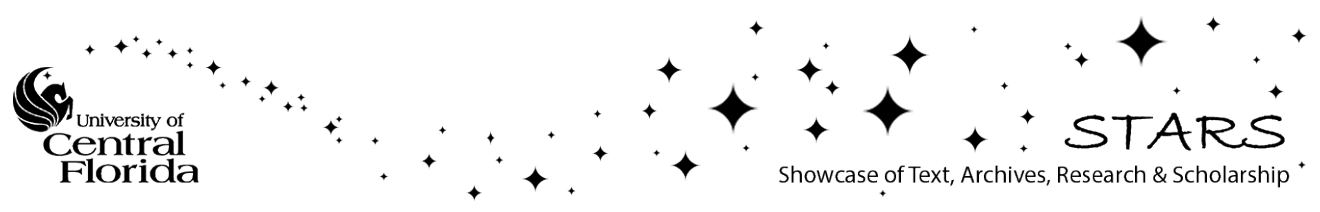




\title{
Crossover of the spectral weight function: A new interpretation of some neutron scattering data
}

\author{
Lee Chow and David Fisher \\ Department of Physics, University of Central Florida, Orlando, Florida 32816
}

(Received 20 July 1987)

\begin{abstract}
Dynamic scaling theory proposed by Halperin and Hohenberg has been successfully applied to small- $q$ neutron scattering data in the past. However, recent constant- $E$-scan neutron scattering data cannot be explained by dynamic scaling theory and a Lorentzian spectral weight function. This seeming contradiction can be removed by a crossover of the spectral weight function from a Lorentzian function to a Gaussian function as $q$ becomes large.
\end{abstract}

The magnetic phase transitions of the $3 d$ ferromagnetic metals $\mathrm{Fe}$ and $\mathrm{Ni}$ have been extensively studied in the past twenty years with a variety of different techniques. ${ }^{1-6}$ Among all those techniques, inelastic neutron scattering has played a major role in the study of the spin dynamics near the critical region as a result of its ability to map out the wave-vector and energy dependence of the spectral weight function $F(q, E)$.

In neutron scattering, the scattering function $S(q, E)$ is given by

$$
S(q, E) \propto \chi(q) F(q, E) \frac{E / k t}{1-\exp (-E / k t)},
$$

where $\chi(q)$ is the static susceptibility and is given by the Ornstein-Zernike form $\chi(q) \propto 1 /\left(\kappa^{2}+q^{2}\right)$ at small wave vector $q . F(q, E)$ is the spectral weight function; at small $q$ as a first-order approximation, it can be taken to be a single Lorentzian and has the following form:

$$
F(q, E)=\frac{1}{(\pi)^{1 / 2} \Gamma(q)} \frac{1}{1+[E / \Gamma(q)]^{2}},
$$

where $\Gamma(q)$ defines a characteristic energy by which the dynamics of the spins are scaled.

According to dynamic scaling theory, ${ }^{7}$ both the spectral weight function $F(q, E)$ and linewidth $\Gamma(q)$ are homogenous functions. In particular, $\Gamma(q)$ can be expressed as

$$
\Gamma(q)=A q^{z} f(\kappa / q),
$$

where $A$ is a material-dependent constant, $z$ is the dynamic exponent, $f(\kappa / q)$ is a scaling function, and $\kappa$ is the inverse correlation length; at $T=T_{c}, f(\kappa / q)=f(0)=1$.

In early neutron scattering experiments ${ }^{1-3}$ at small wave vector $q$, the scattering function $S(q, E)$ was usually sampled by a constant- $q$ scan. In these measurements, the energy dependence of the scattering function is taken at different $q$ values. The energy linewidth $\Gamma(q)$ is then fitted via Eq. (3), and $A$ and $z$ are obtained. This kind of analysis ${ }^{1-3}$ has been quite successful for $q<0.15 \AA^{-1}$, and good agreements were found between measurements and dynamic scaling theory. For example, all the scattering functions can be fitted with a Lorentzian form [Eq. (2)], and the dynamic exponent $z$ was found to be $2.7(2)$ for $\mathrm{Fe}$ (Ref. 1) and 2.46(25) for $\mathrm{Ni}$ (Ref. 2) and agreed with the three-dimensional (3D) Heisenberg exchange model. Because of the steepness of the dispersion curve, it is very difficult to extend the constant $q$ measurement to large $q$. Instead, constant- $E$ scan measurements were taken at large $E$ by Lynn and co-workers. ${ }^{8-11}$ In their experiments, the wave-vector dependence of the scattering function is measured at different $E$ values. At $T=T_{c}$ and, assuming Eq. (2) is valid, $S(q, E)$ can be written as

$$
S(q, E) \propto \frac{1}{q^{2}} \frac{A q^{5 / 2}}{A^{2} q^{5}+E^{2}} \frac{E / k T}{1-\exp (-E / k T)} .
$$

Lynn ${ }^{10}$ has shown that the theoretical relation between the energy $E$ and the peak position $q_{0}$ in the constant $E$ scan experiment is given by

$$
E=3 A q_{0}^{5 / 2},
$$

and the full width at half maximum versus peak position is given by

$$
q_{w}=1.573 q_{0}
$$

When the measurements are compared with the above analysis, they seem to indicate a breakdown of the dynamic scaling analysis. This breakdown causes some controversies between small $q$ (or theory) and large $E$ data. For example, it was found that (1) the material-dependent constants $A$ obtained from Eq. (5) for large $E$ data are less than half the values obtained from Eq. (3) for small $q$ data and (2) the full width at half maximum $q_{w}$ of the constant $E$ scan data at $0.2 \AA^{-1}<q_{0}<0.8 \AA^{-1}$ deviate from the prediction of Eq. (6).

Here, we propose that the spectral weight function $F(q, E)$ crosses over to a Gaussian function as $q$ becomes large. However, we do not expect this hypothesis to hold true as $q$ approaches the zone boundary. ${ }^{12}$ Using this crossover hypothesis, the inconsistencies between the small $q$ and large $E$ measurements can be removed.

Assuming a Gaussian spectral weight function, the scattering function at $T=T_{c}$ is given by

$$
S(q, E) \propto \frac{1}{q^{2}} \frac{1}{A q^{5 / 2}} \exp \left(-\frac{E^{2}}{2 A^{2} q^{5}}\right) \frac{E / k T}{1-\exp (-E / k T)} .
$$

The peak position $q_{0}$ in constant $E$ scan experiments can be found by taking the derivative of Eq. (7) with 
TABLE I. Comparison of $A$ values obtained in different $q$ regions and different equations.

\begin{tabular}{lccc}
\hline \hline & \multicolumn{4}{c}{$A\left({\left.\mathrm{meV} \AA^{5 / 2}\right)}\right.$} & \\
Lost & $\begin{array}{c}\text { Small } q \\
\text { [Eq. (3)] }\end{array}$ & $\begin{array}{c}\text { Large } q \\
\text { [Eq. (5)] }\end{array}$ & $\begin{array}{c}\text { [Eq. (8)] } \\
\text { [Eq. }\end{array}$ \\
\hline $\mathrm{Fe}$ & 130 & 62 & 139 \\
$\mathrm{Ni}$ & 350 & 142 & 318 \\
\hline \hline
\end{tabular}

respect to $q$ and set it equal to zero, which yields

$$
E=\frac{3}{5^{1 / 2}} A q^{5 / 2}
$$

The full width at half maximum $q_{w}$ of the scattering function at constant $E$ can be obtained by setting $S\left(q_{1}, E\right)=S\left(q_{0}, E\right) / 2$ and solving for $q_{1}$. There are two real roots whose difference gives the full width at half maximum $q_{w}$.

$$
q_{w}=0.552 q_{0} \text {. }
$$

When we compare Eq. (8) with neutron data at large $E$, good agreement is found. The best fit of large $E$ data to

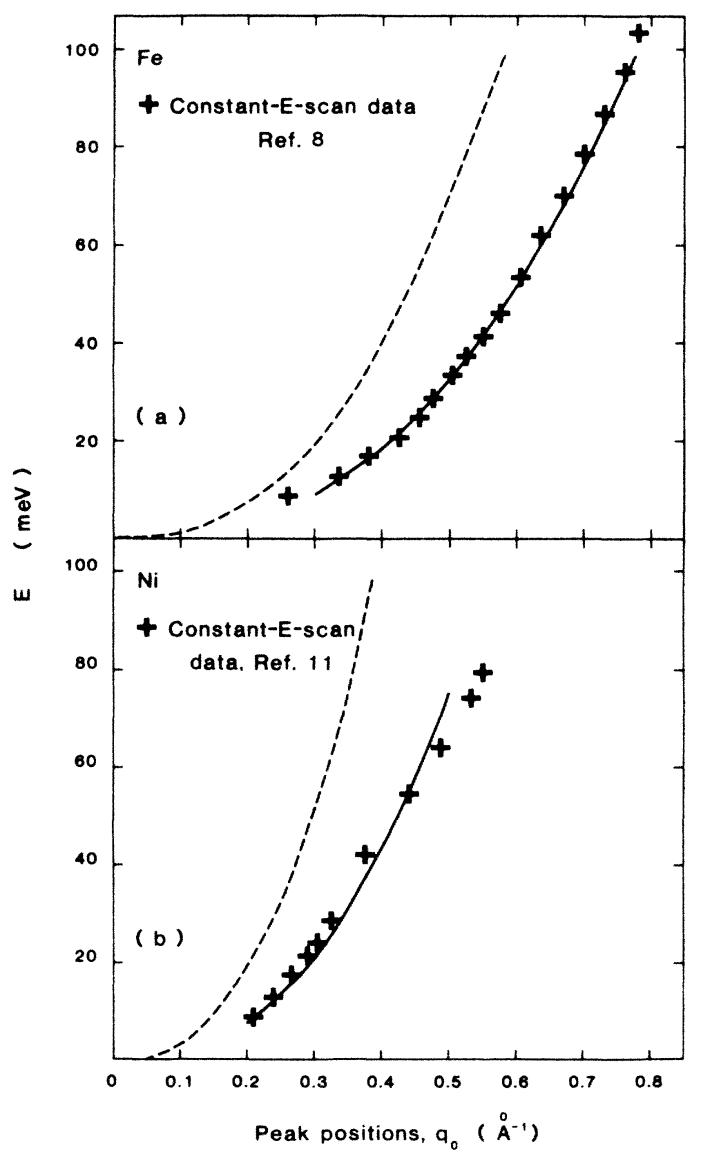

FIG. 1. The energy vs peak positions $q_{0}$ relation for (a) Fe and (b) $\mathrm{Ni}$ at $T \geq T_{c}$. The dashed lines are Eq. (5) with $A$ obtained from small $q$ data. The solid lines are the best fit to Eq. (8).
Eq. (8) yields $A \sim 139 \operatorname{meV} \AA^{5 / 2}$ for $\mathrm{Fe}$ and $A \sim 318$ $\mathrm{meV} \AA^{5 / 2}$ for $\mathrm{Ni}$. These values compare favorably with the values obtained by small- $q$ data as can be seen from Table I and Fig. 1.

In Fig. 2 we plot the predicted behavior of $q_{w}$ vs $q_{0}$ based on the Gaussian and the Lorentzian spectral weight function together with experimental data. This plot makes clear that the deviation of the large $E$ data from $q_{w}=1.573 q_{0}$ can be interpreted as crossover of the spectral weight function to a Gaussian form. Presently, there is no constant $E$ scan data for $q_{0}<0.2 \AA^{-1}$. For $q_{0}>0.2$ $\AA^{-1}(E>8 \mathrm{meV})$, the data already deviate from $q_{w}$ $=1.573 q_{0}$ and approach $q_{w}=0.552 q_{0}$ for $q_{0}>0.7 \AA^{-1}$. In the same graph, a dashed line predicted by the asymptotic renormalization-group (RG) theory ${ }^{13,14}$ is also shown. We will discuss this result later on.

The concept of crossover is not new in the study of critical dynamics. In the hyperfine studies of the spin dynamics $^{15}$ of $\mathrm{Fe}$ and $\mathrm{Ni}$, it was found that at small $q$, the spin dynamics changes over from the 3D Heisenberg model with $z=2.5$ to spin-nonconserved model with $z=2.0$. The concept of using a form other than Lorentzian form for the spectral weight function is also not new. ${ }^{16}$ Hubbard $^{17}$ has shown that for large $\kappa / q$, the spectral weight function will be Lorentzian; as $\kappa / q$ approaches zero, the spectral weight function will have a shape very similar to Gaussian. Marshall and Lovesey ${ }^{18}$ have pointed out that in general the spectral weight function is approximately Gaussian. There are two exceptions to the Gaussian form which occur for (a) very small $q$ at any value of $T$, and (b) at $T \simeq T_{c}$ with $q$ near to an ordering vector. ${ }^{19}$ So it is not surprising that the Lorentzian form only cannot describe the experimental data adequately when $q$ becomes large.

We can qualitatively explain the origin of this crossover of the spectral weight function as follows. In the small-q regime (long-wavelength approximation), each neutron samples a large domain in real space. The time response is an exponential function, so the total differential cross section will have a homogeneously broadened line shape,

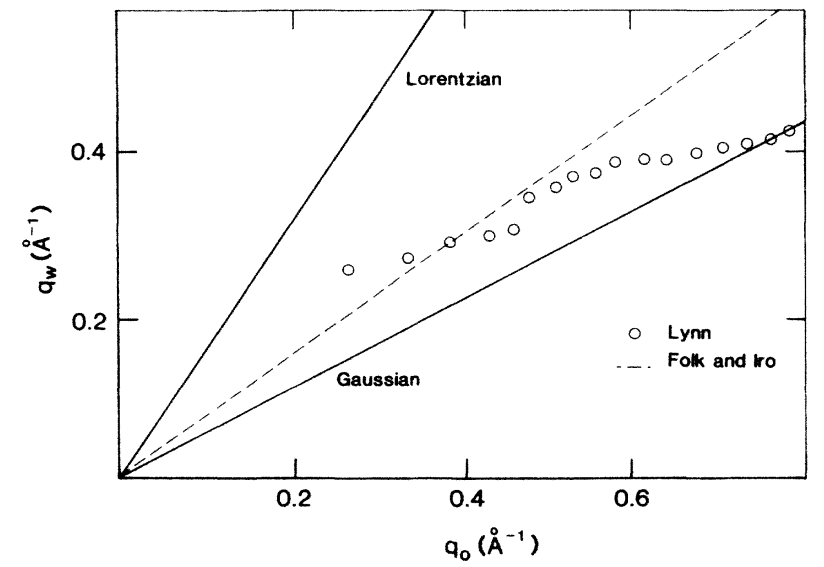

FIG. 2. Comparison of the calculated width $q_{w}$ vs peak positions $q_{0}$ for Lorentzian and Gaussian spectral weight functions. Experimental data on $\mathrm{Fe}$ are also shown here (Ref. 10). The dashed line is predicted by an asymptotic RG theory (Ref. 13). 


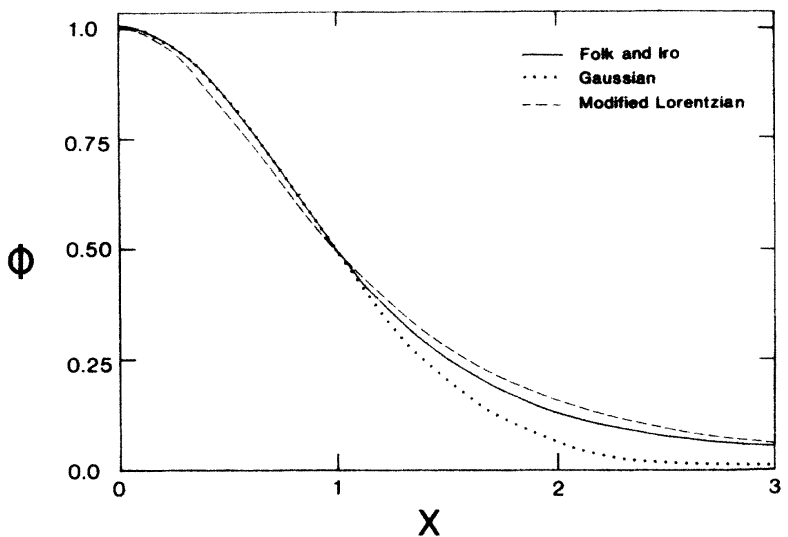

FIG. 3. Comparison of the Gaussian shape function (dotted line), with the modified Lorentzian shape function (dashed line) of Ref. 20, and the asymptotic RG shape function (solid line) of Ref. 13. Note that $x=1.28 \omega / \Gamma$.

i.e., Lorentzian form. As the wave vector $q$ becomes large, each neutron will sample a smaller domain in real space, applying the central limiting theorem, the total differential cross section will reflect an inhomogeneously broadened line shape, namely, a Gaussian.

Recently, Folk and Iro ${ }^{13,14}$ proposed an asymptotic RG theory approach to explain the discrepancies between large- $E$ data and the dynamic scaling theory. They predicted a slope of 0.75 for the $q_{w}$ vs $q_{0}$ plot as shown in Fig. 2 . It will be valuable to extend the constant- $E$ scan experiment to both small-E $\left(q_{0}<0.2 \AA^{-1}\right)$ and large- $E$ $\left(q_{0}>0.8 \AA^{-1}\right)$ regions.

Another approach to this problem was taken by the Shirane and co-workers. ${ }^{20,21}$ They proposed a modified Lorentzian form for the spectral weight function. With a fitting parameter $\alpha=0.1$, they were able to explain the large- $E$ data (see Fig. 12 of Ref. 20). In Fig. 3, we plot the line-shape function $\phi(x)$ based on the asymptotic RG theory (where $x=\omega / A q^{2}$ ), together with the modified Lorentzian and Gaussian form. For small $x$ (large $q$ ), the Gaussian function is identical to $\phi(x)$. For large $x$ (small $q)$, the Gaussian function deviates from $\phi(x)$. This is expected because the Gaussian function is only valid for large $q$.

A final comparison with experiment is shown in Fig. 4. Here we plot the shape of the scattering function $S(q, E)$ at constant $E$ as predicted by the Gaussian, modified Lorentzian, and Lorentzian form at $T=1.02 T_{c}$.

The fits to Lynn's data are also shown in the same figure. We can see that while Lorentzian form cannot describe the data, both Gaussian and modified Lorentzian forms are a considerable improvement over the Lorentzian form.

In conclusion, a crossover of spectral weight function was proposed to explain the constant- $E$ scan neutron data in $\mathrm{Fe}$ and $\mathrm{Ni}$ at large $E$. It was shown that the discrepancy between the measured values of $A$ in the constant- $q$

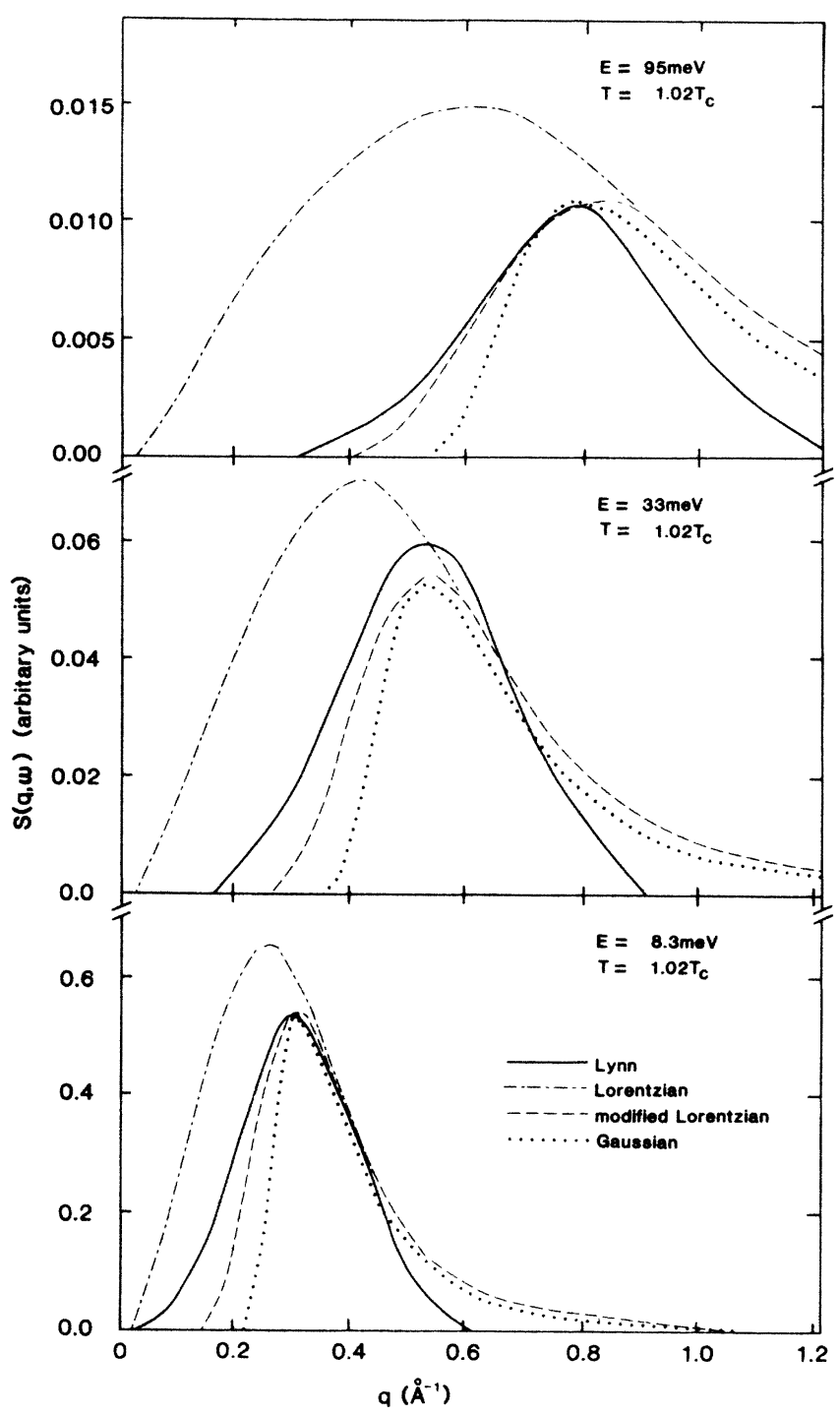

FIG. 4. Comparison of the scattering function $S(q, \omega)$ obtained by Lynn (Ref. 9) with predictions based on modified Lorentzian (Ref. 20) form, and prediction based on Gaussian form. These curves are calculated with $T=1.02 T_{c}$ and $f(\kappa / q)=\exp (-1.83 \kappa / q)+0.43(\kappa / q)^{1 / 2}$ (Ref. 22). We have not taken the instrument resolution into account in our calculation.

scan (small $q$ ) and constant- $E$ scan (large $E$ ) can be removed by our crossover hypothesis. The deviation from a straight line of the $q_{w}$ vs $q_{0}$ data can also be viewed as an indicator of crossover to Gaussian spectral weight function.

We would like to thank Dr. J. W. Lynn and Dr. P. Boni for useful discussion. We also are in debt to Dr. G. S. Collins of Washington State University and Dr. J. Patterson of Florida Institute of Technology for useful discussion. 
${ }^{1}$ M. F. Collins, V. J. Minkiewicz, R. Nathans, L. Passel, and G. Shirane, Phys. Rev. 179, 417 (1969).

${ }^{2}$ V. J. Minkiewicz, M. F. Collins, R. Nathans, and G. Shirane, Phys. Rev. 182, 624 (1969).

${ }^{3}$ V. J. Minkiewicz, Int. J. Magn. 1, 149 (1971).

${ }^{4}$ M. A. Kobeissi, Phys. Rev. B 24, 2380 (1981).

${ }^{5}$ C. Hohenemser, L. Chow, and R. M. Suter, Phys. Rev. B 26, 5056 (1982).

${ }^{6}$ M. Shaham, J. Barak, V. El-Hanany, and W. W. Warren, Jr., Phys. Rev. B 22, 5400 (1980).

${ }^{7}$ P. Hohenberg and B. I. Halperin, Rev. Mod. Phys. 49, 435 (1977); B. I. Halperin and P. Hohenberg, Phys. Rev. 177, 952 (1969).

${ }^{8}$ J. W. Lynn, Phys. Rev. B 11, 2624 (1975).

9 J. W. Lynn, Phys. Rev. B 28, 6550 (1983).

${ }^{10}$ J. W. Lynn, Phys. Rev. Lett. 52, 775 (1984).

${ }^{11}$ J. W. Lynn and H. A. Mook, Phys. Rev. B 23, 198 (1981).

${ }^{12}$ We can not expect the dynamic scaling theory to hold at arbitrarily large $q$. The scaling hypothesis assumes that it is valid under long-wavelength and low-frequency approximation.
${ }^{13}$ R. Folk and H. Iro, Phys. Rev. B 32, 1880 (1985).

${ }^{14}$ R. Folk and H. Iro, Phys. Rev. B 34, 6571 (1986).

${ }^{15}$ R. M. Suter and C. Hohenemser, Phys. Rev. Lett. 41, 705 (1978); L. Chow, R. M. Suter, and C. Hohenemser, ibid. 45, 908 (1980).

${ }^{16}$ In Ref. 10, Lynn has proposed using a function different from a Lorentzian to give a smaller slope. In Ref. 21, Uemura, Shirane, Steinsvoll, and Wicksted also proposed the crossover to Gaussian at high temperatures near the zone boundary.

17 J. Hubbard, J. Phys. C 4, 53 (1971).

${ }^{18}$ W. Marshall and S. W. Lovesey, Thermal Neutron Scattering (Oxford Univ. Press, London, 1971).

${ }^{19}$ In Ref. 17, it was also found that at $T=T_{c}$, as $q$ became large, the line-shape function deviated from the Gaussian form.

${ }^{20}$ J. P. Wicksted, P. Boni, and G. Shirane, Phys. Rev. B 30, 3655 (1984).

${ }^{21}$ Y. J. Uemura, G. Shirane, O. Steinsvoll, and J. Wicksted, Phys. Rev. Lett. 51, 2322 (1983).

22P. Resibois and C. Piette, Phys. Rev. Lett. 24, 514 (1970). 